\title{
POTÊNCIA GERADA EM UM SISTEMA DINÂMICO DE CAPTAÇÃO DE ENERGIA CONTROLADO VIA MÉTODO LQR: COMPARAÇÃO ENTRE EXCITAÇÃO PERIÓDICA E NÃO-IDEAL
}

\section{GENERATED POWER IN AN ENERGY HARVESTER DYNAMIC SYSTEM CONTROLLED VIA LQR METHOD: COMPARISON BETWEEN PERIODIC AND NON-IDEAL EXCITATION}

Estevão Fuzaro de Almeida $^{1}$, Fábio Roberto Chavarette ${ }^{1}$, Douglas da Costa Ferreira ${ }^{2}$

\footnotetext{
${ }^{1}$ Universidade Estadual Paulista Júlio de Mesquita Filho - UNESP, ${ }^{2}$ Universidade Tecnológica Federal do Paraná - UTFPR.

E-mail: estevao.fuzaro@unesp.br
}

RESUMO - A maior parte do controle ativo em sistemas dinâmicos vibracionais é usado para reduzir vibrações. No entanto, o objetivo desta pesquisa é especificamente o uso de vibrações para gerar energia elétrica, de tal forma que a vibração se torne um fenômeno desejado. Dessa forma, a intenção é utilizar o Controle Ideal via Regulador Quadrático Linear (LQR), resultando em maior transdução de energia vibracional para elétrica, através da alteração do tipo de excitação e através de uma análise da estabilidade e dos efeitos que o controle provoca sobre o sistema. O sistema consiste em uma massa-molaamortecedor bimodal com acoplamento piezoelétrico-mecânico que sofre excitação periódica e não-ideal. Objetiva-se determinar qual gera mais energia.

Palavras-chave: Captação de Energia; Controle Ótimo Linear; Excitação Periódica e Não-Ideal.

ABSTRACT - Most of active control in vibrational dynamic systems is used to reduce vibrations. However, the aim of this research is specifically the use of vibrations to generate electrical energy, in such a way that the vibration becomes a desired phenomenon. In this way, the intention is to use the Optimal Control via Linear Quadratic Regulator (LQR), resulting in greater transduction of vibrational energy to electric power, varying the excitation type and performing an analysis of the stability and the effects of control to the dynamic system. The analyzed system is a bimodal mass-spring-damper with piezoelectric couplingmechanic who suffers excitations periodic and non-ideal. This work intends to determine which system generates more electric power.

Keywords: Energy Harvesting; Optimal Linear Control; Periodic and NonIdeal Excitation. 


\section{INTRODUÇÃO}

A demanda por energia elétrica tem crescido ao longo dos anos de acordo com a taxa de crescimento populacional. Nesse cenário, a procura por fontes sustentáveis de energia também vem crescendo. Uma das matrizes de energia sustentável conhecida é a biomassa, mas que para sua geração, provoca impactos ambientais, como a geração de calor e partículas residuais da sua queima. Outro exemplo de geração de energia sustentável é a fusão nuclear, mas que também apresenta desvantagens em termos de alto custo e riscos de manuseio, além dos resíduos radioativos.

Visando, portanto, uma fonte de energia sustentável, segura e eficiente, foram desenvolvidas técnicas que recebem a designação de Captação de Energia (Energy Harvesting, em inglês). As técnicas empregadas buscam captar energia da natureza, como: pequenos gradientes de temperatura, pequenos movimentos de massa e por meio de vibração.

A Captação de Energia é uma alternativa promissora para dispositivos eletrônicos de baixa potência. Além disso, os dispositivos eletrônicos estão se tornando mais eficientes em relação ao consumo de energia, e alguns sensores remotos já podem operar com até $100 \mu W$ (TANG et al., 2013).

O foco deste trabalho são os sistemas mecânicos colhedores de energia baseados em vibração, considerando que para muitas aplicações com sensores remotos e para monitoramento estrutural de integridade, a energia luminosa (de fonte solar) e a energia térmica, podem não estar disponíveis no local de aplicação, gerando uma carência de energia, mesmo que mínima (YOUNGSMAN et al., 2010; SCHLICHTING et al., 2013). Além disso, as captações de energia baseadas em vibração tendem a ter baixos requisitos de manutenção e, além disso, podem ser usadas em ambientes considerados hostis para sistemas mais tecnológicos e complexos; tais ambientes são comumente escolhidos para a alocação desses sensores devido a maior amplitude de vibrações e por esse tipo de colhedor não necessitar de extensos cabos (CHALLA et al., 2008).

Os primeiros modelos de colhedores de energia baseados em vibração apresentam respostas consideradas eficientes apenas se a frequência de vibração de excitação coincidir com a frequência natural do sistema excitado, dando origem ao fenômeno conhecido como ressonância (WU et al., 2006; SARI et al., 2008; EICHHORN et al., 2009; BLARIGAN et al., 2012; TANG et al., 2013). A chamada frequência natural de um corpo é dada pela expressão $\bar{\omega}=\sqrt{\mathrm{k} / \mathrm{m}}$, onde $k$ é a rigidez do sistema e $m$ é sua massa (LIU et al., 2008). Assim, a ressonância ocorre quando a frequência externa de excitação $(\omega)$ é igual à frequência natural do corpo. No entanto, a maior parte da vibração do ambiente tem uma ampla faixa de frequência (HARNE; WANG, 2013; TANG et al., 2013) e se comporta aleatoriamente, por exemplo como acontece durante a vibração das estruturas de construção civil sujeitas à ação eólica (JUNG et al., 2013). Como consequência dessa ampla faixa, o efeito da ressonância é limitado.

Considerando-se as pesquisas desenvolvidas na área $\mathrm{e}$, tendo como objetivo a melhoria da eficiência da captação de energia em sistemas vibracionais, várias soluções foram propostas, sendo uma delas conhecida como multimodal (FERREIRA et al., 2016). A solução multimodal propõe o uso de diferentes vigas a fim de obter melhor aproveitamento das frequências naturais de oscilação do sistema. O efeito da solução multimodal é manter o sistema em ressonância estendida em uma faixa maior de frequência de excitação.

Em relação às formas de excitação do sistema, são encontrados diversos estudos nos quais utilizam-se fontes de excitação periódica, ou seja, que se repetem com o decorrer do tempo. Para esse tipo de análise alteram-se os valores de frequência angular dessas fontes de modo a atingir a ressonância do sistema e obter uma máxima captação de energia para os parâmetros escolhidos. 
Atualmente, as fontes não-ideais se apresentam como uma nova proposta para os sistemas vibracionais (KONONENKO, 1969). A principal característica desse tipo de excitação é que a fonte fornece energia ao sistema, e a resposta dinâmica dele influencia na atuação da fonte. Tais sistemas em que há a interação entre a fonte de energia e o sistema são chamados de sistemas não-ideais (NAYFEH; MOOK, 1979) sendo a fonte chamada de fonte não-ideal. Exemplos de fontes não-ideais são motores elétricos DC escovados, motores de indução, drives com acoplamentos dissipativos ou qualquer tipo de deslizamento dependente de carga etc. (BALTHAZAR et al., 2003; CVETICANIN et al., 2018).

Uma solução adicional ao que foi exposto até aqui, e que busca melhorar a eficiência dos sistemas de captação de energia, dá-se através da utilização de controladores. Como mencionado em trabalhos (HARNE; WANG, 2013; TANG et al., 2013), a maior parte da vibração do ambiente tem comportamento aleatório, variando dentro de uma banda com grande faixa de frequência e apresentando baixa potência. Em um trabalho recente (WANG \& INMAN et al., 2012) é apresentado que a solução mais promissora para melhorar a eficiência dos sistemas de captação de energia baseados na piezeletricidade, através da aplicação de controle ativo, é o Controle Ideal.

Considerando todos os trabalhos citados até aqui, este trabalho propõe a aplicação da teoria de controle moderno via Regulador Quadrático Linear a um sistema de captação de energia com acoplamento piezoelétrico-mecânico submetido a dois tipos de excitações: periódica e não-ideal. São escolhidos parâmetros e condições classificados como instáveis conforme Segundo Método de Lyapunov. O controle ativo LQR será aplicado a fim de viabilizar a construção dos modelos operando com parâmetros instáveis e, posteriormente, comparar a eficácia na captação de energia elétrica entre ambos.

\section{METODOLOGIA}

Os modelos utilizados são baseados em alguns trabalhos dos autores (CHAVARETTE, 2013; ALMEIDA \& CHAVARETTE, 2018; ALMEIDA et al., 2019; FERREIRA et al., 2016). As figuras Figura 1 e Figura 2 apresentam, respectivamente, o modelo do colhedor de energia com excitação periódica e não-ideal.

Figura 1. Sistema com excitação periódica.

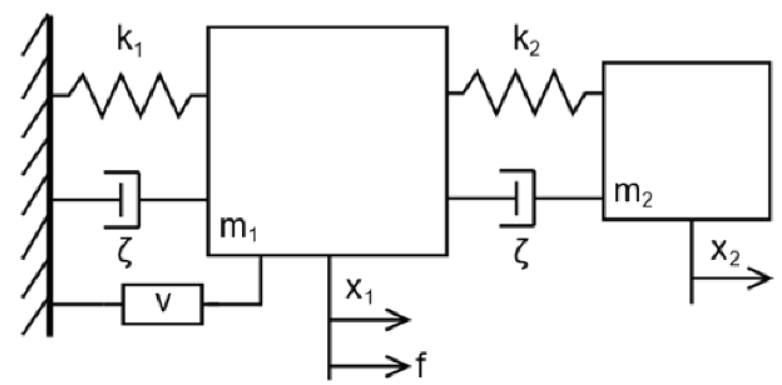

Fonte: Próprios autores.

Figura 2. Sistema com excitação não-ideal.

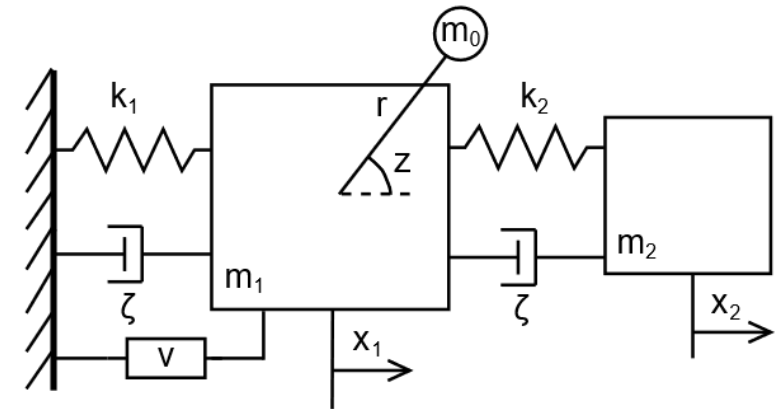

Fonte: Próprios autores.

Os sistemas são compostos por duas massas $\left(m_{1}\right.$ e $\left.m_{2}\right)$ as quais são acopladas à suas respectivas molas $\left(\begin{array}{llll}k_{1} & \mathrm{e} & k_{2}\end{array}\right) \mathrm{e}$ amortecedores ( $\zeta)$; há também um transdutor piezoelétrico $(v)$ responsável pela transdução de energia mecânica para energia elétrica. As rigidezes são distintas para cada massa de prova e para cada comprimento de viga, gerando frequências naturais diferentes. $\mathrm{O}$ amortecimento para cada viga não é alterado, devido ao tipo de material ser o mesmo. Para o caso da Figura 1 há uma fonte de excitação exógena causada por uma força periódica, já para o caso da Figura 2 a fonte de excitação é interna ao sistema, sendo chamada de não-ideal, sendo constituída por uma massa $\left(m_{0}\right)$ 
desbalanceadora a uma distância fixa $(r)$ do eixo de um motor DC, responsável por exercer torques fazendo a massa $\mathrm{m}_{0}$ girar em movimento circular. Devido a essas fontes, há uma resposta vibracional do sistema, causando uma deformação na cerâmica piezoelétrica, gerando uma corrente elétrica que é conduzida através de um circuito resistivo para posterior armazenamento (ERTURK; INMAN, 2011).

\subsection{Descrição matemática}

\subsubsection{Sistema com Excitação Periódica}

O sistema adimensionalizado pela massa que rege o sistema mostrado na Figura 1 é apresentada pelas equações diferenciais nas Equações (1) - (3), nas quais $\zeta$ é a taxa de amortecimento pela massa, $\chi$ é a taxa de acoplamento piezoelétrico-mecânico, $\Lambda$ é a taxa do recíproco da constante de tempo de carregamento capacitivo, $k_{1}$ e $k_{2}$ são as taxas de rigidez e $K$ é a taxa de acoplamento piezoelétrico-elétrico. A excitação periódica externa é descrita por uma taxa de aceleração $f$ e por uma taxa de frequência angular $\omega$. As variáveis dependentes do tempo são $x$ para a taxa de deslocamento de cada viga e $v$ para a taxa de saída de tensão (FERREIRA et al., 2016; ERTURK; INMAN, 2011; ALMEIDA et al., 2019).

$$
\begin{aligned}
& \ddot{x}_{1}+2 \zeta \dot{x}_{1}+k_{1} x_{1}-k_{2}\left(x_{1}-x_{2}\right)-\chi \nu=f \cos (\omega t) \\
& \ddot{x}_{2}+2 \zeta \dot{x}_{2}-k_{2}\left(x_{1}-x_{2}\right)=0 \\
& \dot{\nu}+\Lambda \nu+\mathrm{K}\left(\dot{x}_{1}-\dot{x}_{2}\right)=0
\end{aligned}
$$

Isolando as variáveis temporais e definindo um espaço de estados (no qual $x_{1}=y_{1}, \dot{y}_{1}=y_{2}, x_{2}=y_{3}, \dot{y}_{3}=y_{4}$ e $v=$ $y_{5}$ ), obtêm-se as equações diferenciais apresentadas nas Equações (4) - (8) que descrevem o comportamento dinâmico do sistema periódico.

$$
\begin{aligned}
& \dot{y}_{1}=y_{2} \\
& \dot{y}_{2}=f \cos (\omega t)-2 \zeta y_{2}-k_{1} y_{1}+k_{2}\left(y_{1}-y_{3}\right)+\chi y_{5} \\
& \dot{y}_{3}=y_{4} \\
& \dot{y}_{4}=-2 \zeta y_{4}+k_{2}\left(y_{1}-y_{3}\right) \\
& \dot{y}_{5}=-\Lambda y_{5}-\mathrm{K}\left(y_{2}-y_{4}\right)
\end{aligned}
$$

A Equação (9) é a forma matricial de se representar o sistema de equações apresentados anteriormente, no qual $\boldsymbol{y} \in \mathbb{R}^{n}$ é o vetor de estados, $\mathbf{A} \in \mathbb{R}^{n \times n}$ é a matriz de parâmetros (matriz Jacobiana) e $\boldsymbol{f} \in \mathbb{R}^{n}$ é a excitação exógena.

$\dot{\mathbf{y}}=\mathbf{A y}+\mathbf{f}(t)$

\subsubsection{Sistema com Excitação Não-Ideal}

A descrição matemática adimensional pela massa que rege o sistema mostrado na Figura 2 é apresentada pelas equações diferenciais nas Equações (10) - (13), nas quais os parâmetros adimensionais apresentados anteriormente para o sistema com excitação periódica são os mesmos. Para a fonte não-ideal, $d$ é a taxa de excentricidade da massa desbalanceada e " $a$ " e " $b$ " são as taxas de resistência do torque líquido pelo momento de inércia do motor DC. As variáveis dependentes do tempo são $x$ para a taxa de deslocamento de cada viga, $v$ para taxa de saída de tensão e $z$ para a taxa da posição angular da massa $m_{0}$ (ALMEIDA \& CHAVARETTE, 2018).

$$
\begin{aligned}
& \ddot{x}_{1}+2 \zeta \dot{x}_{1}+k_{1} x_{1}-k_{2}\left(x_{1}-x_{2}\right)-\chi \nu \\
& =d\left(\dot{z}^{2} \cos z+\ddot{z} \sin z\right) \\
& \ddot{x}_{2}+2 \zeta \dot{x}_{2}-k_{2}\left(x_{1}-x_{2}\right)=0 \\
& \ddot{z}+b \dot{z}=r \ddot{x}_{1} \sin z+a \\
& \dot{\nu}+\Lambda \nu+\mathrm{K}\left(\dot{x}_{1}-\dot{x}_{2}\right)=0
\end{aligned}
$$

Já com as variáveis temporais isoladas, define-se um espaço de estados (onde $x_{1}=y_{1}, \dot{y}_{1}=y_{2}, x_{2}=y_{3}, \dot{y}_{3}=y_{4}$, $z=y_{5}, \dot{y}_{5}=y_{6}$ e $\left.v=y_{7}\right)$ e assim se obtém o sistema de Equações (14) - (20) diferenciais e que descrevem o sistema dinâmico nãoideal (ALMEIDA; CHAVARETTE, 2018).

$$
\begin{aligned}
& \dot{y}_{1}=y_{2} \\
& \dot{y}_{2}=\frac{-2 \zeta y_{2}-k_{1} y_{1}+k_{2}\left(y_{1}-y_{3}\right)+\chi y_{7}}{1-d r \sin ^{2} y_{5}}+ \\
& \frac{d\left(y_{6}{ }^{2} \cos y_{5}-b y_{6} \sin y_{5}+a \sin y_{5}\right)}{1-d r \sin ^{2} y_{5}}
\end{aligned}
$$




$$
\begin{aligned}
\dot{y}_{3}= & y_{4} \\
\dot{y}_{4}= & -2 \zeta y_{4}+k_{2}\left(y_{1}-y_{3}\right) \\
\dot{y}_{5}= & y_{6} \\
\dot{y}_{6}= & \frac{-b y_{6}+a+d r y_{6}{ }^{2} \sin y_{5} \cos y_{5}}{1-d r \sin ^{2} y_{5}}+ \\
& \frac{r \sin y_{5}\left[-2 \zeta y_{2}-k_{1} y_{1}+k_{2}\left(y_{1}-y_{3}\right)+\chi y_{7}\right]}{1-d r \sin ^{2} y_{5}} \\
\dot{y}_{7}= & -\Lambda y_{7}-\mathrm{K}\left(y_{2}-y_{4}\right)
\end{aligned}
$$

A Equação (21) é a forma matricial de se representar o sistema de Equações (14) (20). É análogo a Equação (9), mas sem o termo referente à excitação exógena. De acordo com Kononenko (1969), a excitação nesse caso é interna e dependente da resposta dinâmica do sistema.

$$
\dot{\mathbf{y}}=\mathbf{A y}
$$

\subsection{Controle Linear Ótimo - Regulador Quadrático Linear (LQR)}

O desenvolvimento do projeto de Controle Ótimo é baseado nos trabalhos (CHAVARETTE et al., 2009a; CHAVARETTE et al., 2009b; FERREIRA et al., 2015; FERREIRA et al., 2014a; FERREIRA et al., 2014b). Um sistema controlado é governado pela Equação (22), no qual $\boldsymbol{y} \in \mathbb{R}^{n}$ é um vetor de estado, $\mathbf{A} \in \mathbb{R}^{n \times n}$ é uma matriz de parâmetros (matriz Jacobiana), $\mathbf{B} \in \mathbb{R}^{n \times m}$ é uma matriz constante responsável por definir quais variáveis de estado serão controladas e $\boldsymbol{u} \in \mathbb{R}^{m}$ é o vetor de controle, cuja solução tem a forma apresentada pela Equação (23), na qual $\boldsymbol{K}$ é o vetor de realimentação de estado.

$$
\begin{aligned}
\dot{\mathbf{y}} & =\mathbf{A y}+\mathbf{B u} \\
\mathbf{u} & =-\mathbf{K y}
\end{aligned}
$$

Substituindo a Equação (23) na Equação (22), obtém-se o novo sistema controlado, sendo apresentado na Equação (24).

$$
\dot{\mathbf{y}}=(\mathbf{A}-\mathbf{B K}) \mathbf{y}
$$

Definindo-se $\boldsymbol{A}, \boldsymbol{B}, \boldsymbol{Q}$ e $\boldsymbol{R}$ como matrizes constantes, a matriz definida positiva $\boldsymbol{P}$ é obtida através da solução da equação algébrica não-linear de Riccati, apresentada na Equação (25). Com a obtenção de $\boldsymbol{P}$, pode-se calcular o vetor de realimentação $\boldsymbol{K}$ apresentado na Equação (26).

$$
\begin{aligned}
& \mathbf{P A}+\mathbf{A}^{T} \mathbf{P}-\mathbf{P B} R^{-1} \mathbf{B}^{T} \mathbf{P}+\mathbf{Q}=0 \\
& \mathbf{K}=R^{-1} \mathbf{B}^{T} \mathbf{P}
\end{aligned}
$$

Com o vetor de realimentação $\boldsymbol{K}$, o sistema pode ser controlado pelo Regulador Quadrático Linear (LQR). Uma análise complementar que pode ser realizada é através da obtenção dos autovalores da Matriz Jacobiana $(\boldsymbol{A})$ para o sistema sem controle e da Matriz "Jacobiana" Resultante $(\boldsymbol{A}-\boldsymbol{B} \boldsymbol{K})$ para o sistema controlado. Pelo Segundo Método de Lyapunov, se pelo menos um dos autovalores possuir parte real positiva, o sistema pode ser considerado instável; mas se todos os autovalores possuem parte real negativa, o sistema pode ser considerado estável.

\subsection{Potência Adimensional Gerada (RMS)}

A potência adimensional gerada em RMS $(\phi)$ é calculada através da divisão entre a taxa da voltagem de saída ao quadrado $\left(v^{2}\right)$ pela taxa de resistência elétrica $(\Psi)$, como apresentada pela Equação (27). Utilizou-se uma taxa de resistência elétrica $\Psi=0,1$ (ERTURK \& INMAN, 2011; FERREIRA et al., 2016).

$$
\Phi=\frac{\nu_{\mathrm{rms}}^{2}}{\Psi}
$$

\section{RESULTADOS E DISCUSSÃO}

Os resultados foram obtidos utilizando-se os mesmos parâmetros do sistema, de controle e condições iniciais para ambos os casos. Dessa forma, favorece-se a comparação da potência gerada entre os sistemas. 


\subsection{Sistema com Fonte de Excitação Periódica}

\subsubsection{Parâmetros e Condições Iniciais do Sistema}

A Tabela 1 apresenta os parâmetros utilizados que fazem o sistema ter comportamento instável, e a Tabela 2 apresenta as condições iniciais utilizadas para a solução numérica do sistema. Tanto os parâmetros quanto as condições iniciais foram escolhidos arbitrariamente visando a maior captação de energia. A taxa de frequência angular $(\omega)$ é o parâmetro que será alterado a fim de avaliar como a geração de energia é influenciada por ele.

Tabela 1. Parâmetros Instáveis - Excitação Periódica.

\begin{tabular}{ccccccc}
\hline$\zeta$ & $\chi$ & $\mathrm{K}$ & $\Lambda$ & $f$ & $k_{1}$ & $k_{2}$ \\
\hline 0,1407 & 1,30 & 1,07 & 1,10 & 1,00 & 0,60 & 0,20
\end{tabular}

Fonte: Próprios autores.

Tabela 2. Condições Iniciais - Excitação Periódica.

\begin{tabular}{ccccc}
\hline$y_{1}(0)$ & $y_{2}(0)$ & $y_{3}(0)$ & $y_{4}(0)$ & $y_{5}(0)$ \\
\hline 1,30 & 1,10 & 0,50 & 0,20 & 0,98
\end{tabular}

Fonte: Próprios autores.

\subsubsection{Parâmetros de Controle}

Utilizando-se os parâmetros e condições iniciais apresentados nas Tabelas 1 e 2 é possível obter a Matriz Jacobiana $(\boldsymbol{A})$ e, para o controle LQR, conforme apresentado na Equação (28), foram escolhidas as matrizes $\boldsymbol{Q}$ e $\boldsymbol{R}$, e o vetor $\boldsymbol{B}$.

$$
\begin{aligned}
A & =\left[\begin{array}{ccccc}
0 & 1 & 0 & 0 & 0 \\
-0,40 & -0,2814 & -0,20 & 0 & 1,30 \\
0 & 0 & 0 & 1 & 0 \\
0,20 & 0 & -0,20 & -0,2814 & 0 \\
0 & -1,07 & 0 & 1,07 & -1,10
\end{array}\right] \\
Q & =\left[\begin{array}{lllll}
1 & 0 & 0 & 0 & 0 \\
0 & 1 & 0 & 0 & 0 \\
0 & 0 & 1 & 0 & 0 \\
0 & 0 & 0 & 1 & 0 \\
0 & 0 & 0 & 0 & 1
\end{array}\right] \times 10^{-15}, B=\left[\begin{array}{l}
1 \\
1 \\
0 \\
0 \\
1
\end{array}\right], R=[5]
\end{aligned}
$$

São escolhidos valores que são capazes de estabilizar o sistema, alterando a órbita original para uma favorável ao estudo realizado. O vetor $\boldsymbol{B}$ foi determinado através da obtenção do maior valor de controlabilidade possível do sistema. As matrizes $\boldsymbol{Q}$ e $\boldsymbol{R}$ foram escolhidas visando o menor esforço de controle possível. Tal condição faz com que o sistema seja estabilizado sutilmente, de forma com que a captação de energia seja maximizada. Para qualquer valor maior que $1 \times 10^{-15}$ para os elementos da diagonal da matriz $\boldsymbol{Q}$, o sistema é levado ao repouso, o que não é desejável visto que é necessária a vibração para a captação de energia.

\subsubsection{Vetor de Realimentação}

Finalmente, a Equação (29) apresenta o vetor de realimentação para o sistema com excitação periódica obtido solucionando-se as Equações (25) e (26), utilizando as matrizes apresentadas na Equação (28).

$K=\left[\begin{array}{lllll}0,2675 & 0,1865 & -0,3001 & 0,3317 & 0,2070\end{array}\right] \times 10^{-4}$

\subsubsection{Matriz "Jacobiana" Resultante}

Após a obtenção do vetor de realimentação e, com base na Equação (24), pode-se obter a Matriz "Jacobiana" Resultante do sistema controlado, conforme apresentado na Equação (30).

$$
[A-B K]=\left[\begin{array}{ccccc}
-0,00 & 1 & +0,00 & -0,00 & -0,00 \\
-0,40 & -0,28 & -0,20 & -0,00 & 1,30 \\
0 & 0 & 0 & 1 & 0 \\
0,20 & 0 & -0,20 & -0,28 & 0 \\
-0,00 & -1,07 & +0,00 & 1,07 & -1,10
\end{array}\right]
$$

Pode-se notar que não há muita diferença entre as matrizes das Equações (28) e (30), apenas em alguns zeros que passaram a ter sinal negativo, o que indica números pequenos que não foram abrangidos pela quantidade de algarismos significativos apresentados devido aos ganhos pequenos obtidos pelo LQR. Os parâmetros de controle foram escolhidos de forma a alterar sutilmente o sistema de uma órbita instável para uma estável. Tal característica é apresentada a seguir. 


\subsubsection{Estabilidade pelo Segundo Método de Lyapunov}

As Equações (31) - (33) apresentam os autovalores da Matriz Jacobiana sem controle. Considerando que a parte real dos dois últimos autovalores é positiva, o sistema é classificado como instável pelo Segundo Método de Lyapunov.

$$
\begin{aligned}
\lambda_{1,2} & =-0,5465 \pm j 1,2841 \\
\lambda_{3} & =-0,5698+j 0,0000 \\
\lambda_{4,5} & =+0,0000 \pm j 0,3449
\end{aligned}
$$

As Equações (34) - (36) apresentam os autovalores da Matriz "Jacobiana" Resultante $(\boldsymbol{A}-\boldsymbol{B} \boldsymbol{K})$ do sistema controlado. Considerando que a parte real de todos os autovalores é negativa, o sistema é classificado como estável pelo Segundo Método de Lyapunov.

$$
\begin{aligned}
\lambda_{1,2} & =-0,5465 \pm j 1,2841 \\
\lambda_{3} & =-0,5698+j 0,0000 \\
\lambda_{4,5} & =-0,0000 \pm j 0,3449
\end{aligned}
$$

\subsection{Sistema com Fonte de Excitação Não- Ideal}

3.2.1. Parâmetros e Condições Iniciais do Sistema

Os parâmetros em comum tiveram seus valores inalterados a fim de se obter uma comparação fidelizada da geração de potência entre os sistemas. Considerando isso, a Tabela 3 apresenta os parâmetros utilizados exclusivamente para o sistema não-ideal e que também fazem o sistema ter comportamento instável. Já a Tabela 4 apresenta as condições iniciais utilizadas.

Tabela 3. Parâmetros Instáveis - Excitação Não-Ideal.

\begin{tabular}{cccc}
\hline$a$ & $b$ & $d$ & $r$ \\
\hline 0,50 & 0,01 & 0,01 & 1,00
\end{tabular}

Fonte: Próprios autores.
Tabela 4. Condições Iniciais - Excitação NãoIdeal.

\begin{tabular}{ccccccc}
\hline$y_{1}(0)$ & $y_{2}(0)$ & $y_{3}(0)$ & $y_{4}(0)$ & $y_{5}(0)$ & $y_{6}(0)$ & $y_{7}(0)$ \\
\hline 1,30 & 1,10 & 0,50 & 0,20 & 0,60 & 0,30 & 0,98
\end{tabular}

Fonte: Próprios autores.

\subsubsection{Parâmetros de Controle}

Utilizando-se os parâmetros e condições iniciais apresentados nas Tabelas 3 e 4 é possível obter a Matriz Jacobiana $(\boldsymbol{A})$ e, para o controle LQR, conforme apresentado na Equação (37), foram escolhidas as matrizes $\boldsymbol{Q}$ e $\boldsymbol{R}$, e o vetor $\boldsymbol{B}$.

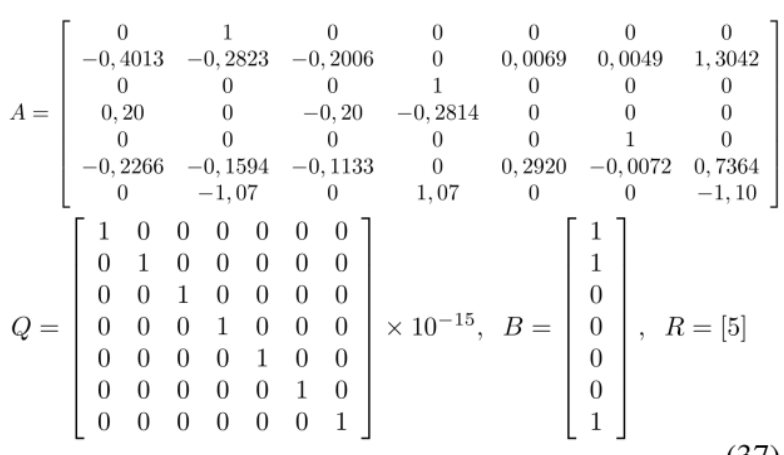

O procedimento de escolha de tais parâmetros é análogo ao que já fora realizado com o sistema com fonte de excitação periódica.

\subsubsection{Vetor de Realimentação}

A Equação (38) apresenta o vetor de realimentação para o sistema com excitação não-ideal obtido solucionando-se as Equações (25) e (26), utilizando as matrizes apresentadas na Equação (37).

$$
\begin{aligned}
K=\left[\begin{array}{llll}
0,2094 & 1,1349 & 0,1837 & -0,1396 \\
& -1,4243 & -2,6262 & -0,2779
\end{array}\right]
\end{aligned}
$$

\subsubsection{Matriz "Jacobiana" Resultante}

Após a obtenção do vetor de realimentação e, com base na Equação (24), pode-se obter a Matriz "Jacobiana" Resultante do sistema controlado, conforme apresentado na Equação (39). 


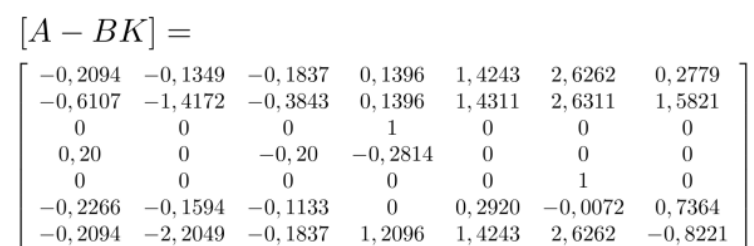

(39)

3.2.5. Estabilidade pelo Segundo Método de Lyapunov

As Equações (40) - (44) apresentam os autovalores da Matriz Jacobiana sem controle. Considerando que a parte real do terceiro, quarto e quinto autovalores é positiva, o sistema é classificado como instável pelo Segundo Método de Lyapunov.

$$
\begin{aligned}
\lambda_{1,2} & =-0,5464 \pm j 1,2851 \\
\lambda_{3} & =+0,5330+j 0,0000 \\
\lambda_{4,5} & =+0,0001 \pm j 0,3448 \\
\lambda_{6} & =-0,5591+j 0,0000 \\
\lambda_{7} & =-0,5523+j 0,0000
\end{aligned}
$$

As Equações (45) - (49) apresentam os autovalores da Matriz "Jacobiana" Resultante $(\boldsymbol{A}-\boldsymbol{B} \boldsymbol{K})$ do sistema controlado. Considerando que a parte real de todos os autovalores é negativa, o sistema é classificado como estável pelo Segundo Método de Lyapunov, o que significa que o controle foi eficiente.

$$
\begin{aligned}
\lambda_{1,2} & =-0,5464 \pm j 1,2851 \\
\lambda_{3,4} & =-0,0001 \pm j 0,3448 \\
\lambda_{5} & =-0,5591+j 0,0000 \\
\lambda_{6} & =-0,5523+j 0,0000 \\
\lambda_{7} & =-0,5330+j 0,0000
\end{aligned}
$$

\subsection{Potência gerada com base na taxa de} frequência angular da fonte de excitação periódica

Solucionando-se computacionalmente os sistemas obtidos pelas Equação (9) e Equação (24) (acrescida do termo referente a excitação periódica) através do Método de Dormand-Prince (Runge-Kutta de 4a Ordem com Passo Variável), obteve-se um gráfico, Figura 3, que apresenta a potência gerada em função da taxa de frequência angular da fonte de excitação periódica. Pode-se observar a banda de frequência relativa à ressonância do sistema estudado.

Figura 3. Taxa de potência (RMS) em função da taxa de frequência angular da fonte de excitação periódica.

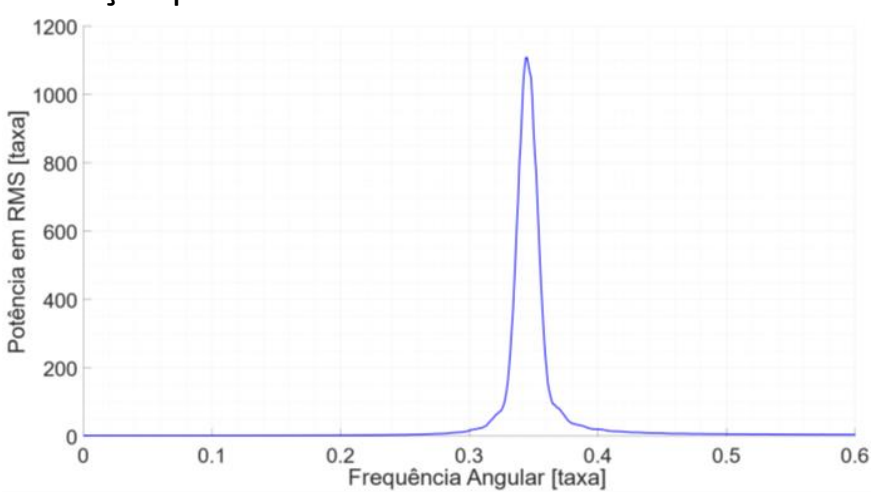

(a) Original

Fonte: Próprios autores.

(b) Ampliado

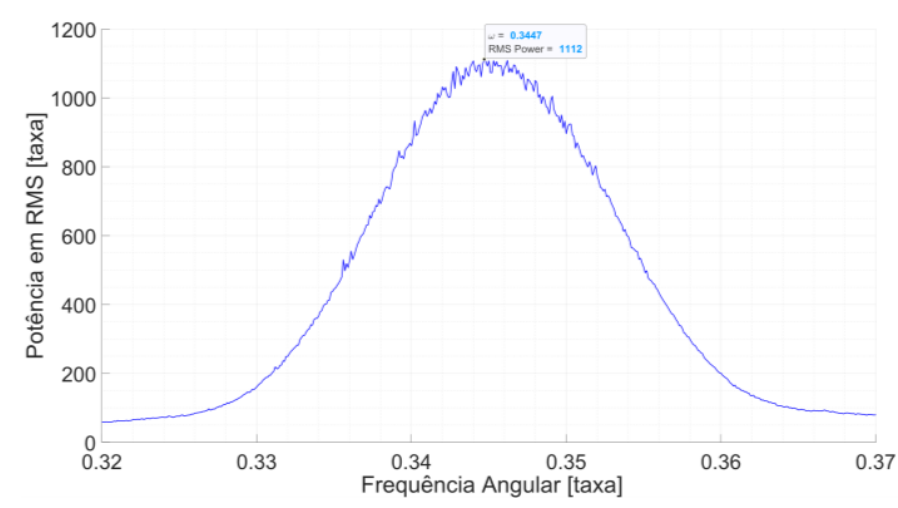

Fonte: Próprios autores.

Com base nesse gráfico, foram escolhidas três taxas de frequências angulares diferentes que abrangessem principalmente a banda de ressonância. Com isso, pode-se realizar uma comparação com o sistema não-ideal e verificar de fato qual deles gera maior potência. As taxas de frequências angulares escolhidas foram: $\omega=0,25$ (antes da ressonância), $\omega=0,45$ (após a ressonância) e $\omega=0,3447$ (na ressonância).

\subsection{Comparação entre os sistemas de captação de energia}

\subsubsection{Taxa de frequência angular $\omega=0,25$}

Primeiramente, analisou-se o sistema controlado antes da ressonância. Os planos 
de fase referentes ao comportamento dinâmico da Massa 1 são apresentados na Figura 4; os da Massa 2, na Figura 5; e o da tensão, na Figura 6.

Figura 4. Planos de fase da Massa 1 $(\omega=0,25)$.

(a) Deslocamento $\times$ Tempo

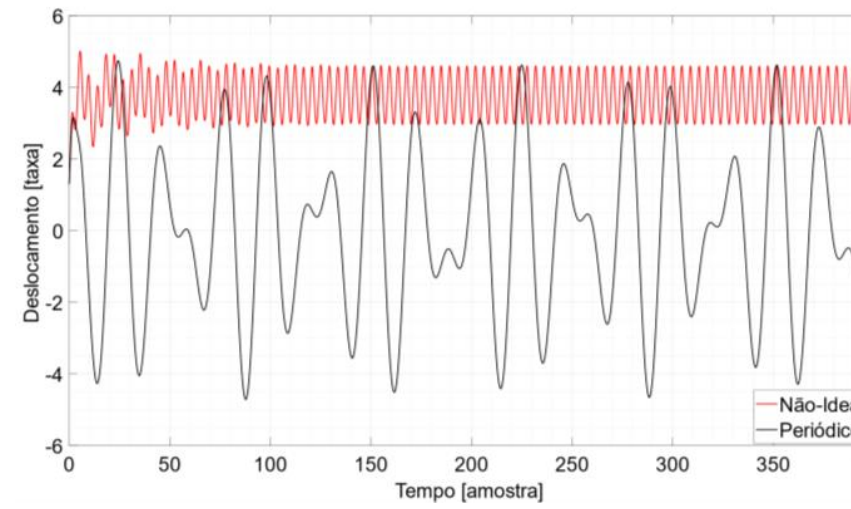

(b) Velocidade $\times$ Tempo

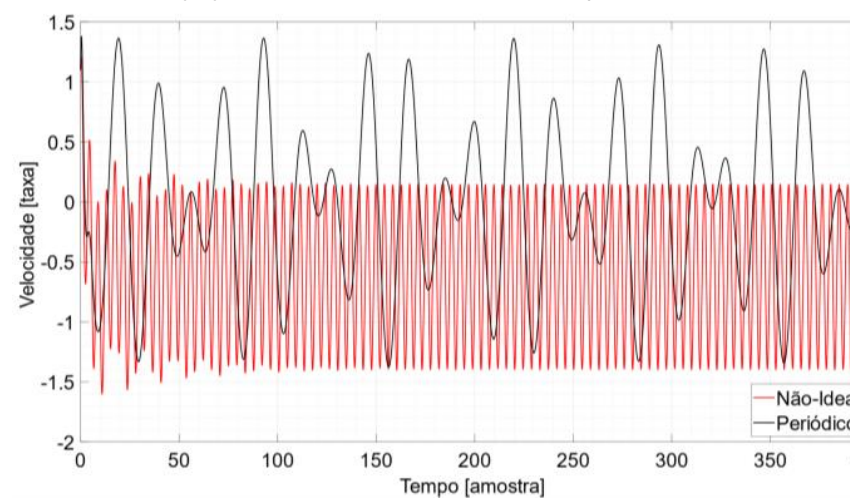

(c) Deslocamento $\times$ Velocidade

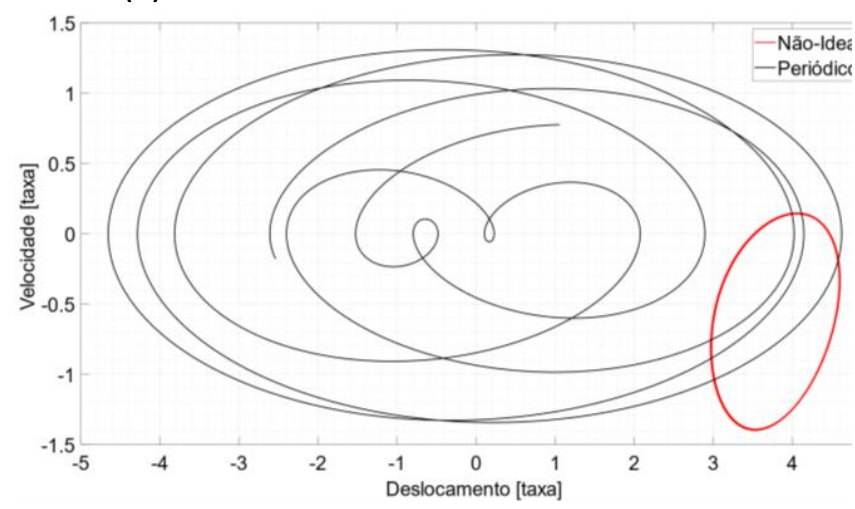

(d) Plano Tridimensional

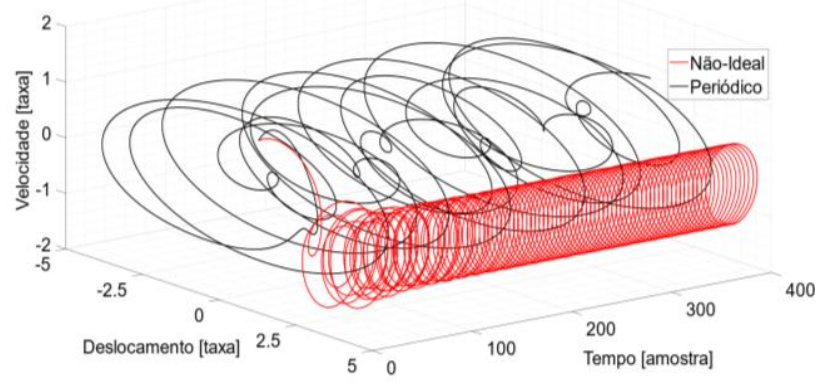

Fonte: Os autores.

Figura 5. Planos de fase da Massa 2 $(\omega=0,25)$.

(a) Deslocamento $\times$ Tempo

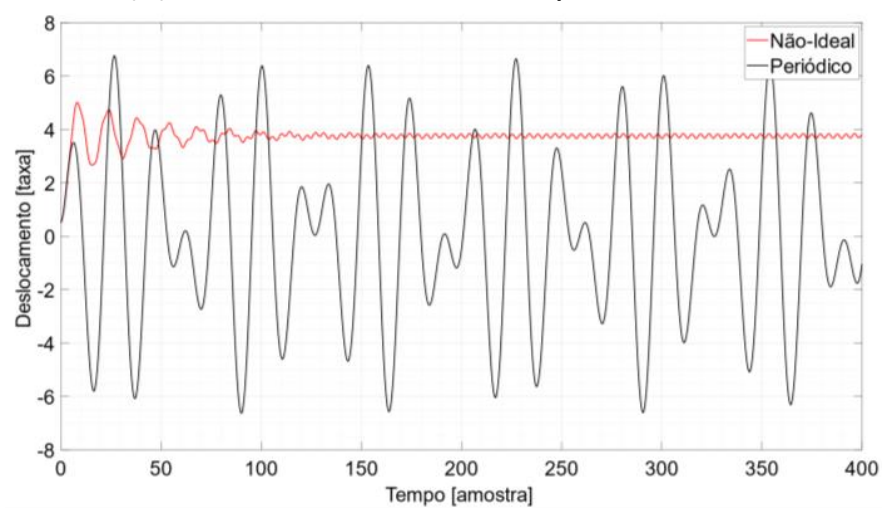

(b) Velocidade $\times$ Tempo

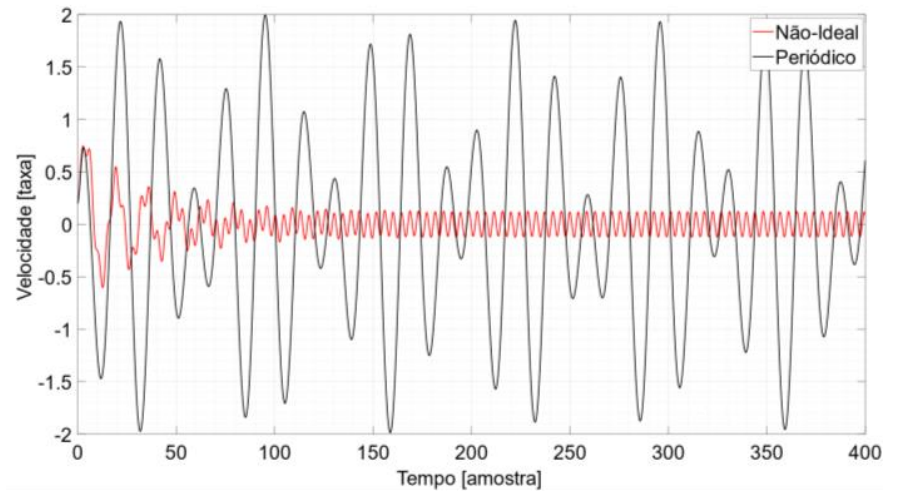

(c) Deslocamento $\times$ Velocidade

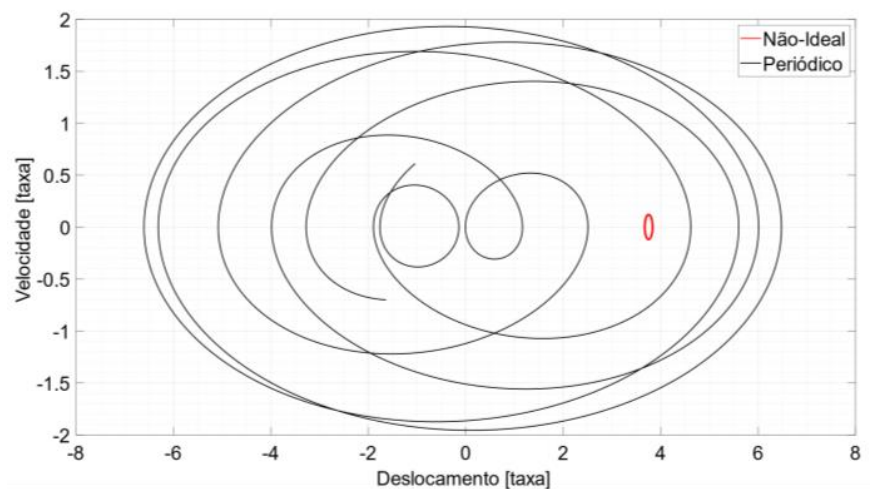


(d) Plano Tridimensional

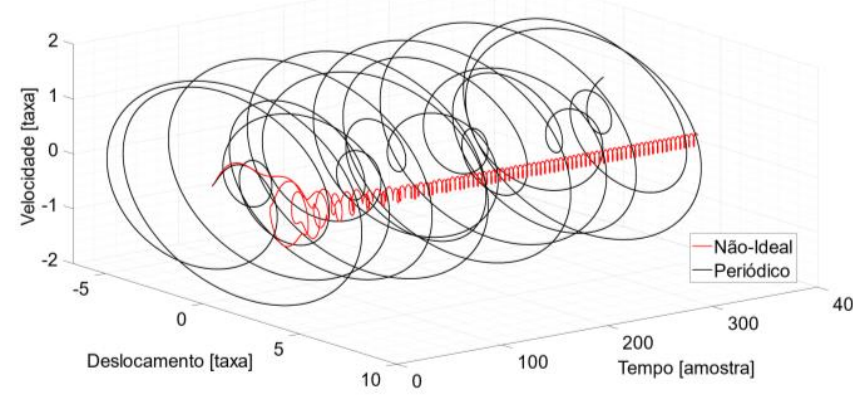

Fonte: Próprios autores.

Figura 6. Taxa de Tensão $\times$ Tempo $(\omega=$ $0,25)$.

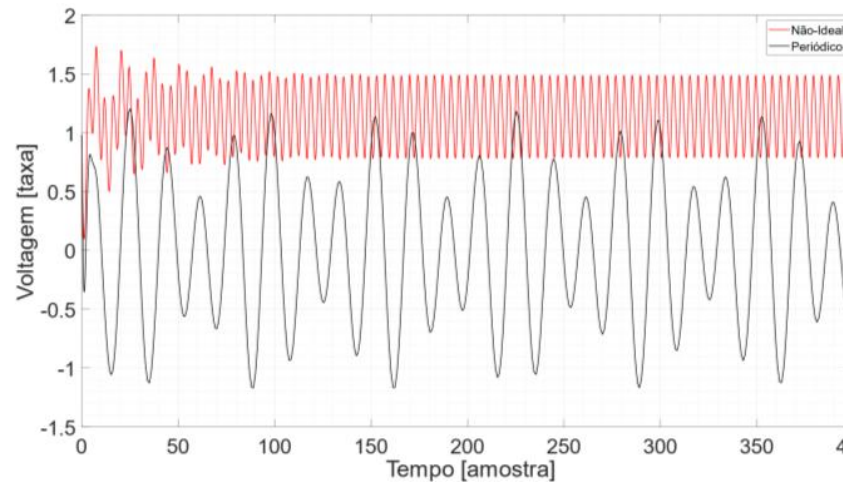

Fonte: Próprios autores.

Nota-se que o sistema não-ideal apresenta menores amplitudes de deslocamento e velocidade em comparação com o sistema periódico. A tensão do sistema periódico apresenta maior amplitude que o sistema não-ideal, sendo que a potência em RMS gerada para tal caso, utilizando-se a Equação (27), é apresentada na Tabela 5, no final dos Resultados e Discussão.

\subsubsection{Taxa de frequência angular $\omega=0,45$}

Em sequência, analisou-se o sistema controlado após a ressonância. Os planos de fase da Massa 1 são apresentados na Figura 7; os da Massa 2, na Figura 8; e o da tensão, na Figura 9.
Figura 7. Planos de fase da Massa 1 $(\omega=0,45)$.

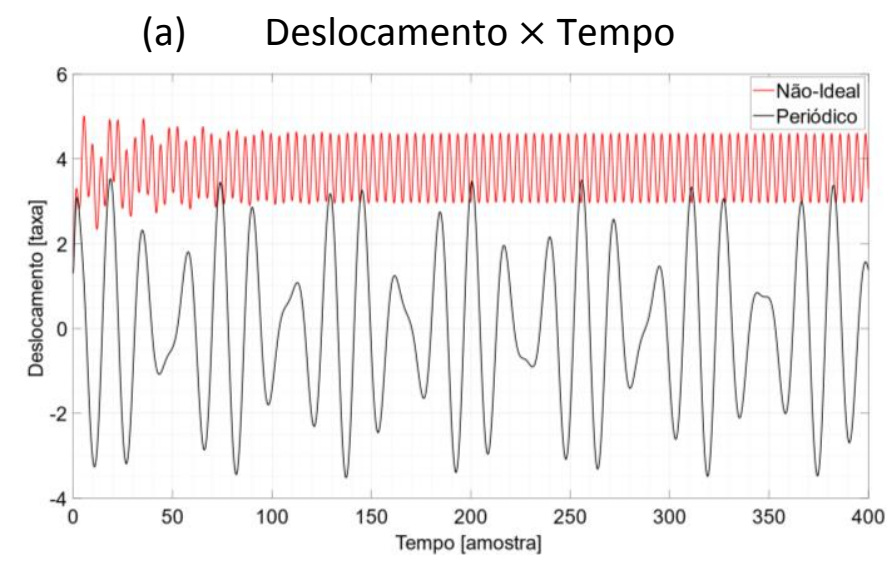

(b) Velocidade $\times$ Tempo

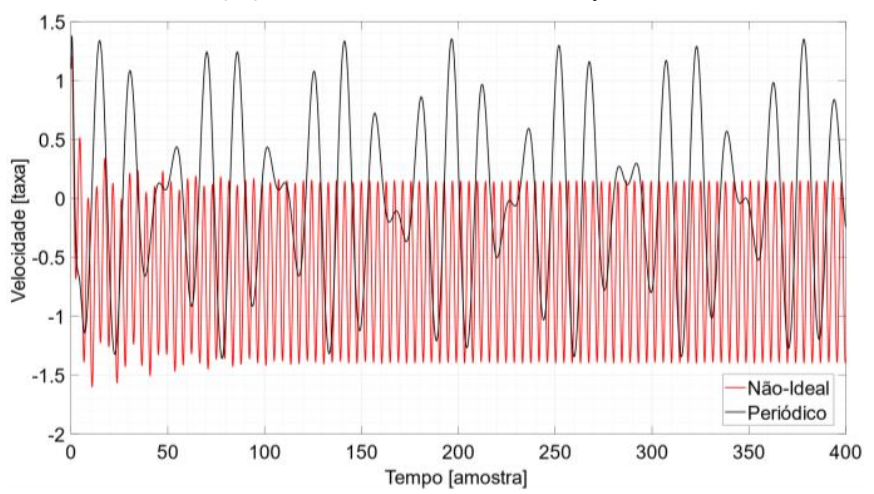

(c) Deslocamento $\times$ Velocidade

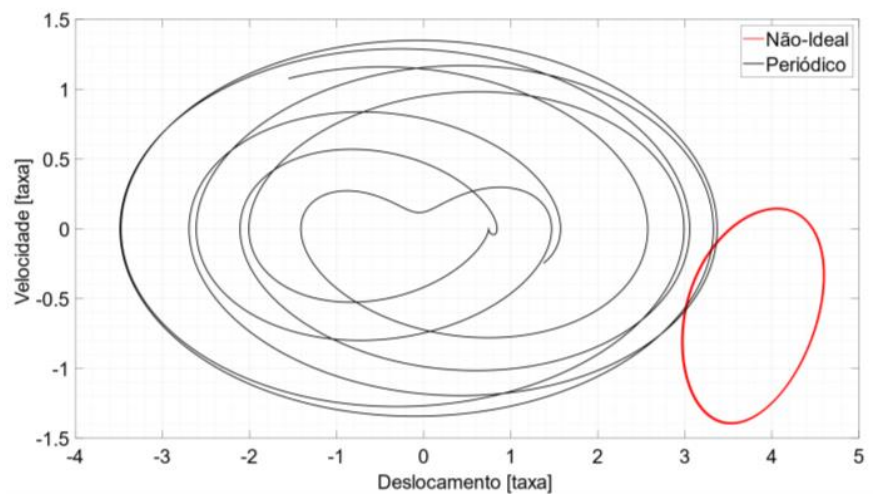

(d) Plano Tridimensional

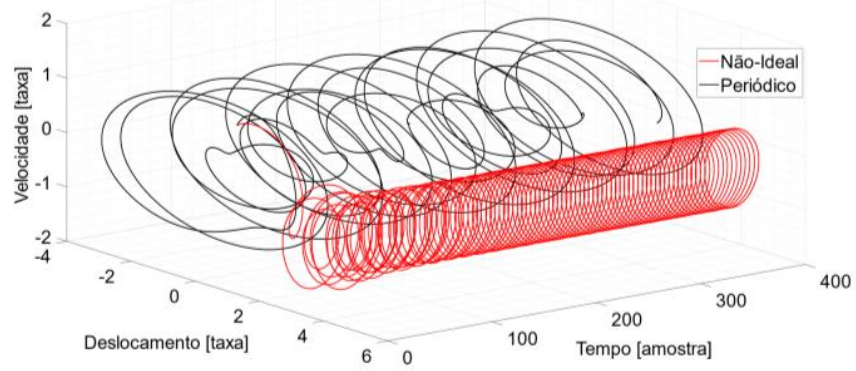

Fonte: Os autores. 
Figura 8. Planos de fase da Massa 2 $(\omega=0,45)$.

(a) Deslocamento $\times$ Tempo

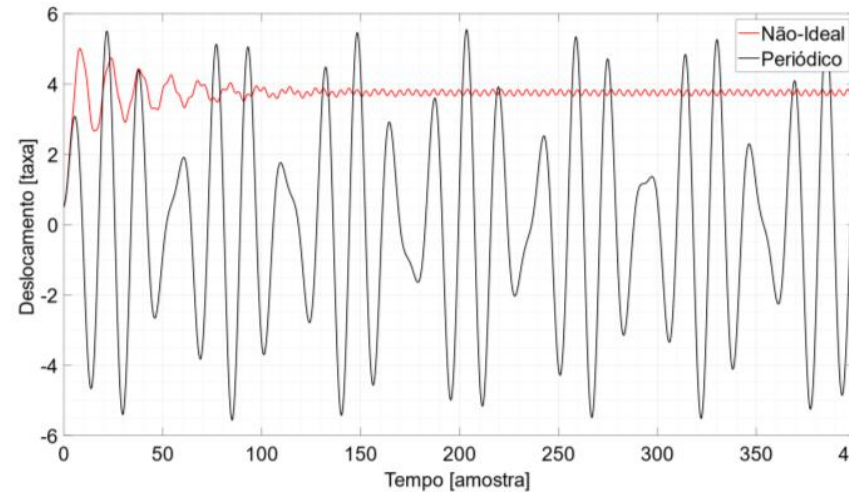

(b) Velocidade $\times$ Tempo

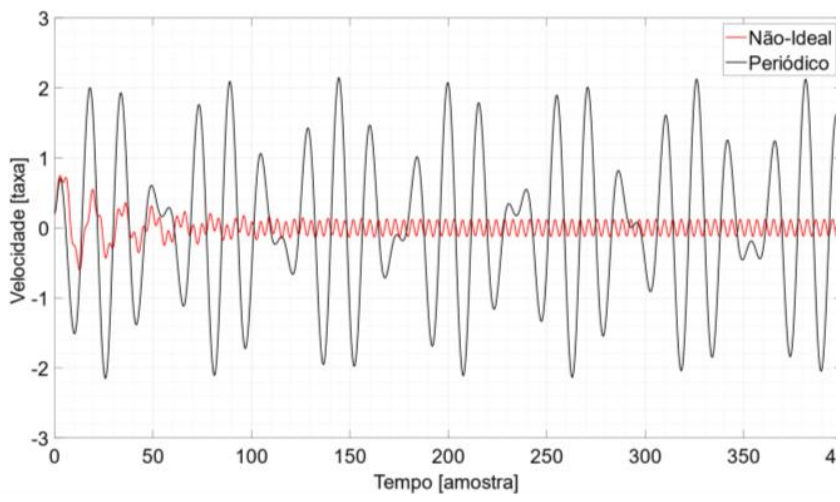

(c) Deslocamento $\times$ Velocidade

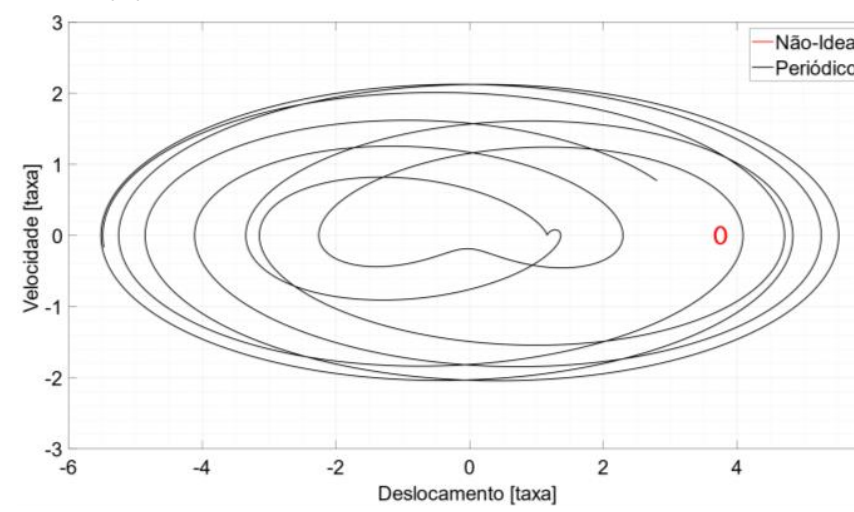

(d) Plano Tridimensional

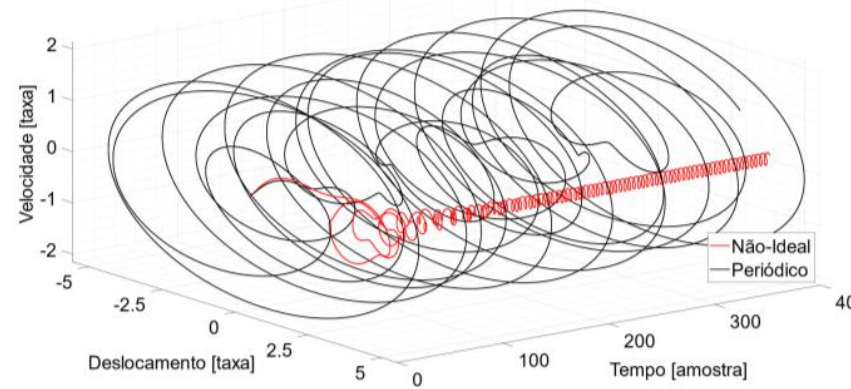

Fonte: Os autores.
Figura 9. Taxa de Tensão $\times$ Tempo $(\omega=$ $0,45)$.

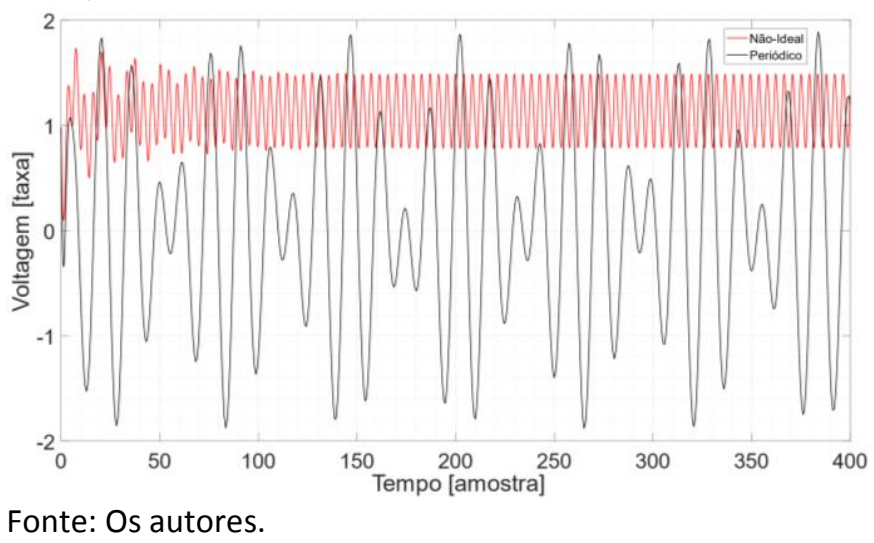

Novamente nota-se que o sistema não-ideal apresenta menores amplitudes de deslocamento e velocidade e maior amplitude de tensão em comparação com o sistema periódico. E, utilizando-se novamente a Equação (27), pode-se obter a taxa de potência em RMS, conforme Tabela 5 apresentada no final dos Resultados e Discussão.

3.4.3. Taxa de frequência angular $\omega=0,3447$

Por último, analisou-se o sistema controlado na ressonância. Os planos de fase da Massa 1 são apresentados na Figura 10; os da Massa 2, na Figura 11; e o da tensão, na Figura 12.

Figura 10. Planos de fase da Massa 1 $(\omega=0,3447)$.

(a) Deslocamento $\times$ Tempo

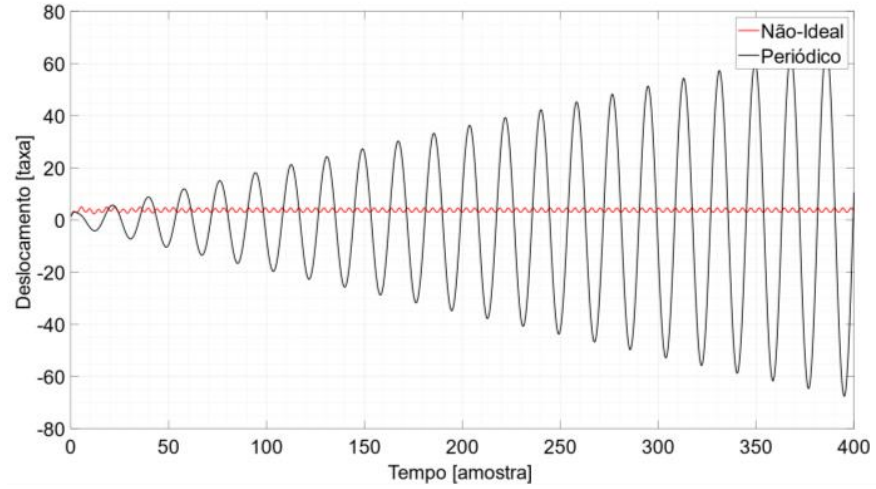


(b) Velocidade $\times$ Tempo

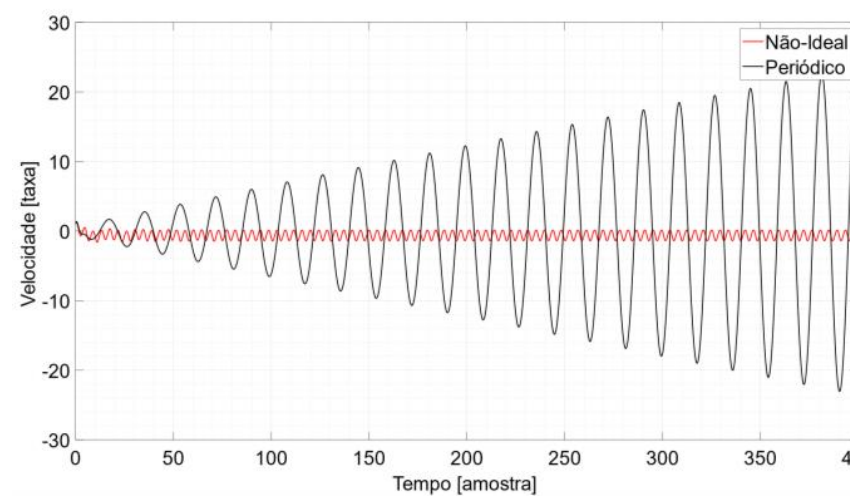

(c) Deslocamento $\times$ Velocidade

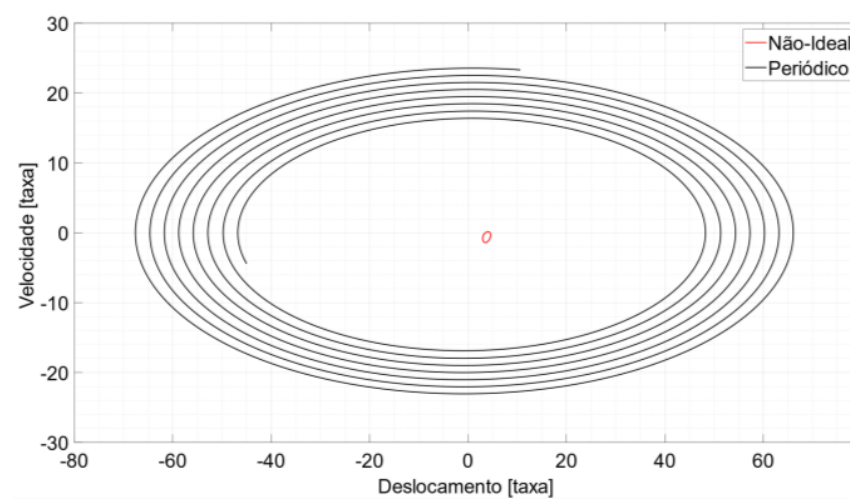

(d) Plano Tridimensional

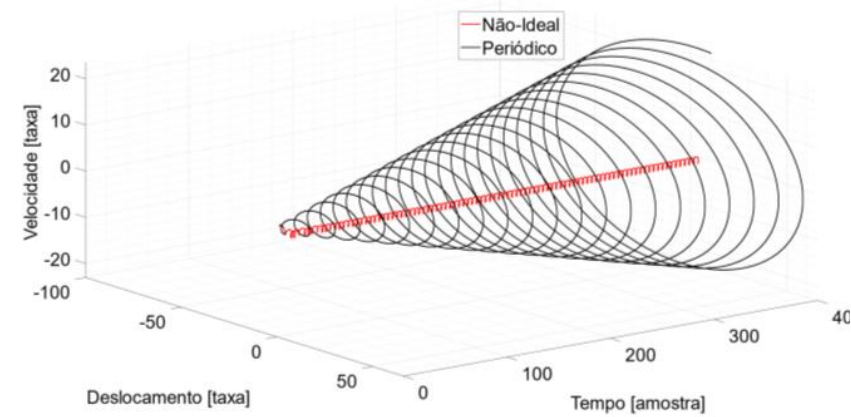

Fonte: Os autores.

Figura 11. Planos de fase da Massa 2 $(\omega=0,3447)$.

(a) Deslocamento $\times$ Tempo

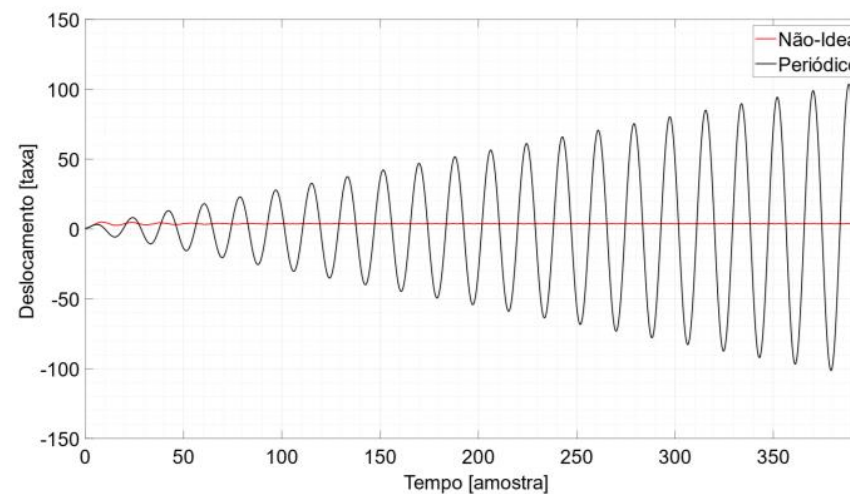

(b) Velocidade $\times$ Tempo

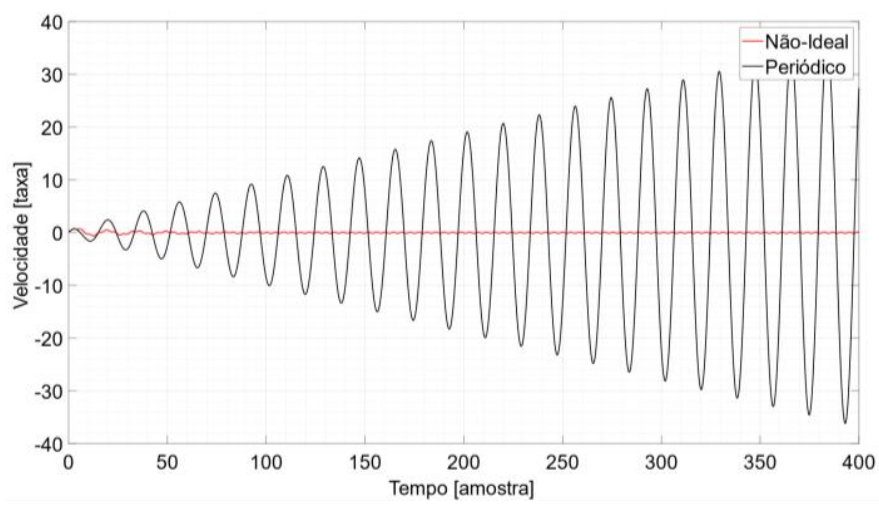

(c) Deslocamento $\times$ Velocidade

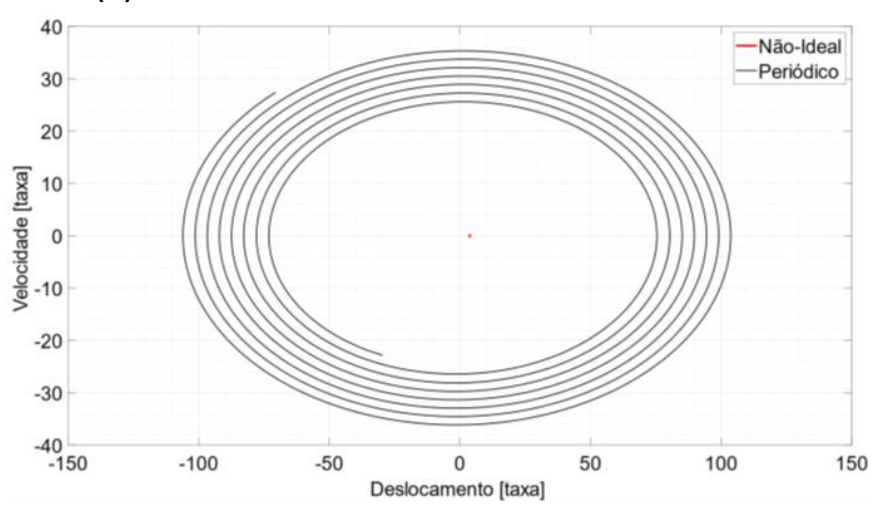

(d) Plano Tridimensional

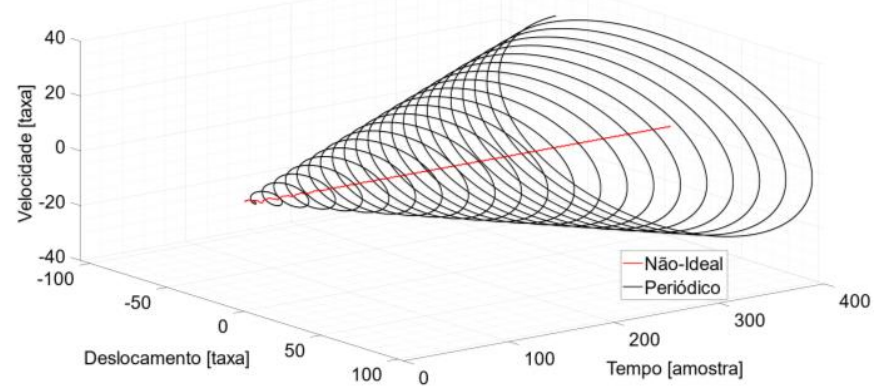

Fonte: Os autores.

Figura 12. Taxa de Tensão $\times$ Tempo $(\omega=0,3447)$.

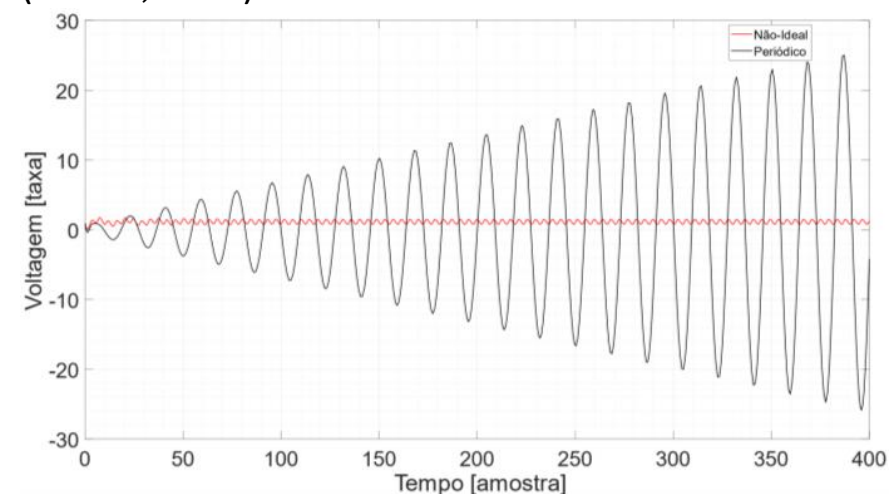

Fonte: Os autores. 
Nesse caso em específico, nota-se uma amplitude sempre crescente em todos os planos de fase do sistema periódico. Tal efeito é exclusivo da ação da ressonância, mesmo com o sistema controlado, a tendência é de que a órbita seja cada vez maior, o que acaba por inviabilizar a construção de um modelo com tais comportamentos. Como efeito comparativo, pode-se obter quanto de taxa de potência em RMS é gerada pela Equação (27). As potências calculadas estão presentes na Tabela 5 do tópico subsequente.

\subsubsection{Taxas de potência em RMS geradas}

A Tabela 5 apresenta as taxas de potência em RMS calculadas pela Equação (27).

Tabela 5. Taxas de potência em RMS.

\begin{tabular}{lcccc}
\hline & \multicolumn{3}{c}{ Periódico } & Não-Ideal \\
\hline Freq. Ang. $(\omega)$ & 0,25 & 0,3447 & 0,45 & - \\
\hline Pot. (RMS) $(\Phi)$ & 3,6740 & 1112,3 & 7,3830 & 12,7171 \\
Fonte: Próprios autores. & & &
\end{tabular}

Já era conhecido que o controlador é capaz de maximizar a captação de energia antes e depois da banda de ressonância do sistema com fonte de excitação periódica (ALMEIDA et al., 2019), melhorando a eficiência do sistema.

Em complemento, nota-se um aumento na geração de potência, comparando o sistema não-ideal com o periódico, de cerca de $276 \%$ para quando $\omega=0,25$ e um aumento de $72 \%$ para quando $\omega=0,45$. Para $\omega=0,3447$, observa-se que o sistema periódico apresenta um aumento de cerca de $8646 \%$, porém, como discutido previamente, a construção de um modelo em ressonância é uma incerteza, como visto pelos planos de fase das Figuras 10, 11 e 12.

\section{CONSIDERAÇÕES FINAIS}

Com os resultados obtidos, pode-se concluir que o controle sempre será vantajoso para qualquer taxa de frequência angular do sistema com fonte de excitação periódica e para o sistema com fonte de excitação não-ideal, visto que a órbita é alterada, estabilizando-se os sistemas.

Conclui-se também que o sistema com fonte de excitação não-ideal gera maior potência, quando comparado com o sistema com fonte de excitação periódica, somente fora da zona ressonante. Na zona ressonante, apesar do sistema periódico gerar maior potência, não é possível sua construção, visto que as órbitas se mantêm crescentes com o passar do tempo, visto que quanto mais próximo a zona ressonante, mais difícil se torna o trabalho realizado pelo controlador, elevando os custos com o esforço computacional e mecânico dos atuadores.

\section{AGRADECIMENTOS}

Os autores agradecem ao laboratório SisPLEXOS, local no qual o projeto foi desenvolvido. Os autores agradecem a Faculdade de Engenharia de Ilha Solteira da Universidade Estadual Paulista (FEIS/UNESP) pelo suporte técnico e acadêmico. $E$ os autores agradecem a Fundação de Amparo à Pesquisa do Estado de São Paulo, Processos No. 2017/03829-7 e 2018/26690-7, pelo suporte moral e financeiro.

\section{REFERÊNCIAS}

ALMEIDA, E. F.; CHAVARETTE, F. R. Captação de energia e análise de estabilidade em um transdutor piezelétrico com excitação nãoideal. In: ASSOCIAÇÃO BRASILEIRA DE ENGENHARIA E CIÊNCIAS MECÂNICAS $A B C M$. Anais do XXV Congresso Nacional de Estudantes de Engenharia Mecânica - CREEM 2018. 2018. v. 3, p. 194-200. Disponível em: https://abcm.org.br/uploadsAAnais\%20do\%20XXV\%20CREEM\%20vol.3.pd f

ALMEIDA, E. F.; CHAVARETTE, F. R.; FERREIRA, D. C. Optimal linear control applied in a energy harvesting dynamic system with periodic excitation. In: ASSOCIAÇÃO BRASILEIRA DE ENGENHARIA E CIÊNCIAS MECÂNICAS - ABCM. 25th ABCM International Congress of Mechanical 
Engineering - COBEM 2019. 2019. Disponível em: https://abcm.org.br/anais/cobem.

BALTHAZAR, J. M. et al. An overview on nonideal vibrations. Meccanica, Kluwer Academic Publishers, v. 38, n. 6, p. 613-621, 2003.

https://doi.org/10.1023/A:1025877308510

BLARIGAN, L. V.; DANZL, P.; MOEHLIS, J. A broadband vibrational energy harvester. Applied Physics Letters, AIP, v. 100, n. 25, p. 253904, 2012. https://doi.org/10.1063/1.4729875

CASTRUCCI, P. B. de L.; BITTAR, A. Controle automático. [S.I.]: Grupo Gen-LTC, 2000.

CHALLA, V. R. et al. A vibration energy harvesting device with bidirectional resonance frequency tunability. Smart Materials and Structures, IOP Publishing, v. 17, n. 1, p. 015035, 2008. https://doi.org/10.1088/0964-

\section{$\underline{1726 / 17 / 01 / 015035}$}

CHAVARETTE, F. Control design applied to a non-ideal structural system with behavior chaotic. International Journal of Pure and Applied Mathematics, v. 86, p. 487-500, 07 2013. https://doi.org/10.12732/ijpam.v86i3.3

CHAVARETTE, F. R. et al. On non-linear dynamics and control designs applied to the ideal and non-ideal variants of the FitzhughNagumo (FN) mathematical model. Communications in Nonlinear Science and Numerical Simulation, Elsevier, v. 14, n. 3, p. 892-905, 2009a. https://doi.org/10.1016/i.cnsns.2007.10.016

CHAVARETTE, F. R. et al. On non-linear dynamics and an optimal control synthesis of the action potential of membranes (ideal and non-ideal cases) of the Hodgkin-Huxley (HH) mathematical model. Chaos, Solitons \& Fractals, Elsevier, v. 39, n. 4, p. 1651-1666, 2009b.

https://doi.org/10.1016/i.chaos.2007.06.016
CVETICANIN, L.; ZUKOVIC, M.; BALTHAZAR, J. $M$. Dynamics of mechanical systems with non-ideal excitation. [S.I.]: Springer, 2018. https://doi.org/10.1007/978-3-319-54169-3

EICHHORN, C. et al. A piezoelectric harvester with an integrated frequency-tuning mechanism. Power MEMS, p. 45-48, 2009.

ERTURK, A.; INMAN, D. J. Broadband piezoelectric power generation on highenergy orbits of the bistable duffing oscillator with electromechanical coupling. Journal of Sound Vibration, v. 330, p. 23392353, May 2011. Disponível em: http://adsabs.harvard.edu/abs/2011JSV...330.2339E. https://doi.org/10.1016/j.jsv.2010.11.018

FERREIRA, D. C. et al. Multimodal energy harvesting efficiency enhancement via linear matrix inequalities control driven. Proceeding Series of the Brazilian Society of Computational and Applied Mathematics, v. 4, n. $1, \quad 2016$. https://doi.org/10.5540/03.2016.004.01.001 $\underline{2}$

FERREIRA, D. C.; CHAVARETTE, F. R.; PERUZZI, $\mathrm{N}$. J. Linear matrix inequalities control driven for non-ideal power source energy harvesting. Journal of Theoretical and Applied Mechanics, v. 53, n. 3, p. 605-616, $2015 . \quad$ https://doi.org/10.15632/itampl.53.3.605

FERREIRA, D. da C.; CHAVARETTE, F. R.; PERUZZI, N. J. Non-linear energy harvesting system efficiency comparison from periodic to non-ideal excitation. International Journal of Pure and Applied Mathematics, Academic Publications, Ltd., v. 92, n. 5, p. 745-755, 2014a.

https://doi.org/10.12732/ijpam.v92i5.9

FERREIRA, D. da C.; CHAVARETTE, F. R.; PERUZZI, N. J. Optimal linear control driven for piezoelectric non-linear energy harvesting on nonideal excitation sourced. In: TRANS 
TECH PUBL. Advanced Materials Research. [S.I.], 2014b. v. 971, p. 1107-1112. https://doi.org/10.4028/www.scientific.net/ AMR.971-973.1107

HARNE, R. L.; WANG, K. A review of the recent research on vibration energy harvesting via bistable systems. Smart materials and structures, IOP Publishing, v. 22, n. 2, p. 023001, 2013. https://doi.org/10.1088/0964$\underline{1726 / 22 / 2 / 023001}$

JUNG, H.-J. et al. A hybrid electromagnetic energy harvesting device for low frequency vibration. In: INTERNATIONAL SOCIETY FOR OPTICS AND PHOTONICS. Active and Passive Smart Structures and Integrated Systems 2013. [S.I.], 2013. v. 8688 , p. 868811 . https://doi.org/10.1117/12.2010014

KONONENKO, V. O. Vibrating systems with a limited power supply. [S.I.]: Iliffe, 1969.

LIU, J.-Q. et al. A mems-based piezoelectric power generator array for vibration energy harvesting. Microelectronics Journal, Elsevier, v.39, n. 5, p. 802-806, 2008. https://doi.org/10.1016/i.mejo.2007.12.017

NAYFEH; MOOK. Nonlinear oscillations. [S.I.]: New York, NY: Wiley-Interscience, 1979.

SARI, I.; BALKAN, T.; KULAH, H. An electromagnetic micro power generator for wideband environmental vibrations. Sensors and Actuators A: Physical, Elsevier, v. 145, p. 405-413, 2008. https://doi.org/10.1016/i.sna.2007.11.021

SCHLICHTING, A.; FINK, E.; GARCIA, E. A lowloss hybrid rectification technique for piezoelectric energy harvesting. Smart Materials and Structures, IOP Publishing, v. 22, n. 9, p. 095028, 2013. https://doi.org/10.1088/0964$\underline{1726 / 22 / 9 / 095028}$
TANG, L.; YANG, Y.; SOH, C. K. Broadband vibration energy harvesting techniques. In: Advances in energy harvesting methods. [S.I.]: Springer, 2013. p. 17-61. https://doi.org/10.1007/978-1-4614-5705-

$\underline{32}$

WANG, Y.; INMAN, D. J. A survey of control strategies for simultaneous vibration suppression and energy harvesting via piezoceramics. Journal of Intelligent Material Systems and Structures, Sage Publications Sage UK: London, England, v. 23, n. 18, p. 2021-2037, 2012. https://doi.org/10.1177/1045389X12444485

WU, W. -J. et al. Tunable resonant frequency power harvesting devices. In: INTERNATIONAL SOCIETY FOR OPTICS AND PHOTONICS. Smart Structures and Materials 2006: Damping and Isolation. [S.I.], 2006. v. 6169, p. 61690A. https://doi.org/10.1117/12.658546

YOUNGSMAN, J. M. et al. A model for an extensional mode resonator used as a frequency-adjustable vibration energy harvester. Journal of Sound and Vibration, Elsevier, v. 329, n. 3, p. 277-288, 2010. https://doi.org/10.1016/i.jsv.2009.09.011 